\title{
Assessment of Frequency of Errors in Conventional Panoramic Radiographs
}

\author{
Abedeera Jayasuriya Seena Patabedige Nileema*, Thasanthan Loganathan, \\ Dissanayake Mudiyanselage Janaka Nuwan \\ Department of Radiography/Radiotherapy, Faculty of Allied Health Sciences, University of Peradeniya, Peradeniya, Sri Lanka
}

\section{Email address:}

spnjayasooriya@gmail.com (A. J. S. P. Nileema), thasanth@pdn.ac.lk (T. Loganathan), janakanu@gmail.com (D. M. J. Nuwan)

${ }^{*}$ Corresponding author

\section{To cite this article:}

Abedeera Jayasuriya Seena Patabedige Nileema, Thasanthan Loganathan, Dissanayake Mudiyanselage Janaka Nuwan. Assessment of Frequency of Errors in Conventional Panoramic Radiographs. International Journal of Dental Medicine. Vol. 2, No. 2, 2016 , pp. 5-8. doi: $10.11648 / j . i j d m .20160202 .11$

Received: October 25, 2016; Accepted: November 8, 2016; Published: December 10, 2016

\begin{abstract}
Although use of conventional panoramic systems leads to degrade the image quality, it is still common in Sri Lanka. The objective of the study was to determine the frequency of errors occurred during Ortho Pantomo Graph (OPG) and to minimize the further occurrence. Two hundred and fifty OPGs were collected from Department of Radiology, Dental Hospital (Teaching) Peradeniya (DHTP). Quality of 250 OPGs was evaluated by one radiologist and a radiographer. Evaluators assessed all 250 radiographs for presence of errors in categories including patient preparation, patient positioning, patient motion, exposure, handling and processing. The frequency of each error was recorded. Further, number of repeated radiographs was also recorded. There were only $22(9 \%)$ error-free OPGs. $228(91 \%)$ radiographs had errors. 6\% of OPGs were unacceptable and had to be re-taken. The frequencies of errors in conventional OPGs were relatively high in this study with patient positioning errors being the most frequent error.
\end{abstract}

Keywords: Radiography, Ortho Pantomo Graph, Quality Control

\section{Introduction}

Dental practitioners frequently rely on OPG for variety of medical and surgical applications related to dentition and related structures owing to its high clinical efficacy [1]. OPG allows the dentist to view the entire dentition and related structures in a single film [2-7]. These are among difficult to produce as the anatomical region of interest (ROI) is more susceptible movements [8]. As well as the complexity of bony details of the ROI lead to difficulty in image interpretation [9]. Nevertheless, the final aim of OPG is to produce high diagnostic quality radiographs to provide better patient care through an accurate diagnosis.

Technological advancements have been led to development of computed and digital imaging systems in imaging field of medicine and dentistry. However, use of conventional OPG imaging systems is still common in Sri Lanka. These systems include a conventional panoramic equipment with screen-type cassettes, films and manual or automatic film processing facility. Generally, the occurrence of errors related to processing and handling are higher with a conventional system in spite of normal occurrence of patient positioning errors which could occur in any system.

With more congested clinics in Sri Lanka, the time spent for taking radiographs has become an important factor determining quality of the radiographs and eventually the care provided to the patients. As well as, majority of OPGs produced in clinics suffer from number of errors. Therefore, generally some amounts of radiographs are being repeated in clinics due to these facts. But, at the time of writing this article no any study was found in literature, which was conducted in Sri Lanka. Therefore, in the present study our aims were to assess the frequency of errors found in a collected sample of conventional panoramic radiographs and to give a clue to minimize the probability of these errors in future. It would be beneficial to the dental imaging field practitioners to deliver their skills efficiently. 


\section{Materials and Methods}

Ethical clearance was taken from the ethical review committee, Faculty of Allied Health Sciences, University of Peradeniya and the permission was taken from the Dean, Faculty of Dental Sciences, University of Peradeniya. A total of 250 OPGs were collected from the Department of Radiology, DHTP, between June 2013 to September 2013. From all OPG referrals over 18 years of age paediatric, emergency and referrals on public holidays were excluded.

All radiographs were produced using the same panoramic radiographic equipment (Planmeca Proline XC, Helsinki, Finland) which is operating at $60-80 \mathrm{kVp}, 4-10$ $\mathrm{mA} \mathrm{DC}$ and exposure time 2.5- 18 seconds for panoramic according to the manufacture's guidelines [10]. Flat cassettes, the films of $15 \times 30 \mathrm{~cm}$ and $12.5 \times 30 \mathrm{~cm}$ sizes are used with this equipment and the image magnification in panoramic is a constant 1.2 [10]. All the radiographs used in the study were processed manually by using the conventional manual film processor at the DHTP.

All the radiographs were produced by well experienced radiographers at DHTP who usually perform the examination in clinics. The radiographs were evaluated by well expertise one maxillo facial radiologist and a radiographer. The same viewing box and the same viewing environment of DHTP were facilitated for both evaluators at DHTP. The original OPGs were evaluated after the processing.

Errors including patient preparation, patient positioning, patient motion, exposure, processing and handling were assessed. Patient preparation errors included failure to remove removable dentures, nasal rings, and any piercing in ROI which would cause artifacts. Patient positioning errors included tongue is not placed against roof of the mouth; palatoglossal air space, head tipped down, head tipped up, head turned right or left, tooth too posterior to bite block, tooth too anterior to bite block, cervical spine slumped, bite block not used. Over exposure, under exposure and double exposure were considered as possible exposure errors. Developer staining, fixer staining, scratch marks, finger prints were included in the film processing and handling errors category. Data were collected on specially formed data sheets and frequency of each error was recorded and number of repeated radiographs also was recorded. The results were presented using descriptive statistics.

\section{Results}

Panoramic radiographs of 250 patients were evaluated. Only $22(9 \%)$ out of 250 radiographs were error-free and rest $228(91 \%)$ radiographs had errors of which $16(6 \%)$ OPGs were unacceptable and had to be re-taken. The number of errors in each radiograph varied between 1 to 4 , where majority of the images had $2 \leq$ errors. $35 \%$ of images had only one error followed by $40 \%, 10 \%$ and $6 \%$ of images had two errors, three errors and four errors respectively.

Table 1. Errors observed on the 250 OPG radiographs examined in the study.

\begin{tabular}{lll}
\hline Error & Frequency of errors & Percentage \% \\
\hline Patient Positioning & 220 & $88 \%$ \\
Processing \& Handling & 132 & $53 \%$ \\
Patient Preparation & 33 & $13 \%$ \\
Exposure & 15 & $6 \%$ \\
Patient Motion & 0 & 0 \\
\hline
\end{tabular}

Errors due to malpositioning of the patient were observed in $220(88 \%)$ images being the most frequent error. There was no any image suffered from the error due to patient motion during the exposure. Exposure errors presented in 15 OPGs $(6 \%)$ being the least frequent error. This was the only reason for an image to be retaken in the present study since it was difficult to observe the anatomy of the ROI due to exposure error. Frequency distribution of types of errors in the study sample of images is shown in table 1 .

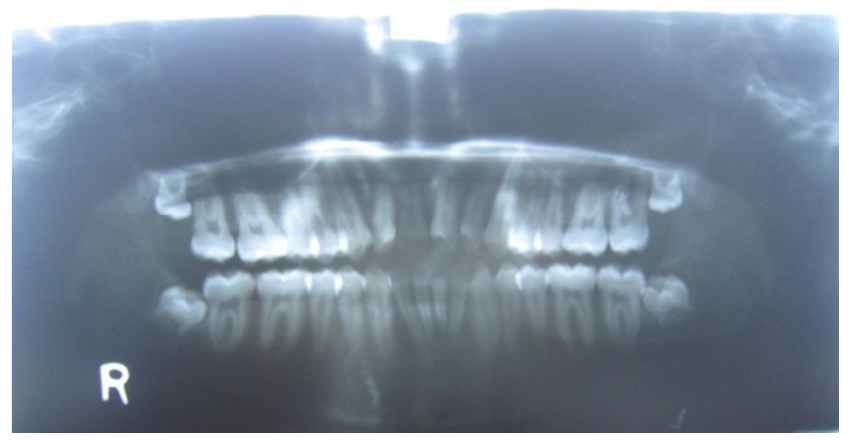

Figure 1. The exposure is too high (over-exposure), reducing the overall contrast of the image.

Only $39(16 \%)$ images were free of positioning errors. But most among these images were with other types of errors. Total of 266 patient positioning errors were encountered in the study sample of images. The failure to position the tongue against the palate was the most common error observed $(86,32 \%)$ being the most common positioning error whereas head tipped up presented in $13(5 \%)$ images being the least common positioning error. Table 2 shows the frequency distribution of patient positioning errors in the study sample of images.

Table 2. Frequency distribution of patient positioning errors in the study sample of images.

\begin{tabular}{lll}
\hline Positioning Error & Number & Frequency \\
\hline Tongue is not placed against roof of the & 86 & $32 \%$ \\
mouth; palatoglossal air space & 54 & $20 \%$ \\
Cervical spine slumped & 45 & $17 \%$ \\
Tooth too posterior to bite block & 32 & $12 \%$ \\
Head tipped down & 23 & $9 \%$ \\
Bite block not used & 13 & $5 \%$ \\
Head tipped up & 13 & $5 \%$ \\
Head turned right/left & 0 & 0 \\
Tooth too anterior to bite block & & \\
\hline
\end{tabular}




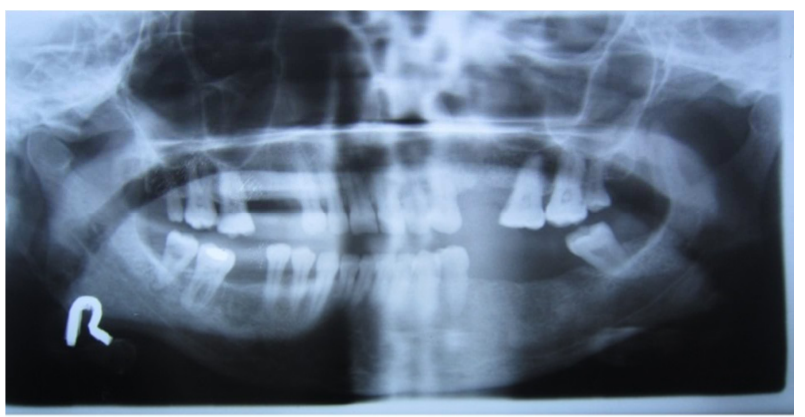

Figure 2. Panoramic radiograph showing patient failed to place the tongue against the hard palate demonstrating a dark band (Palato-glossal air space) over the maxillary teeth and film handling artifatcs over the region of interest (ROI).

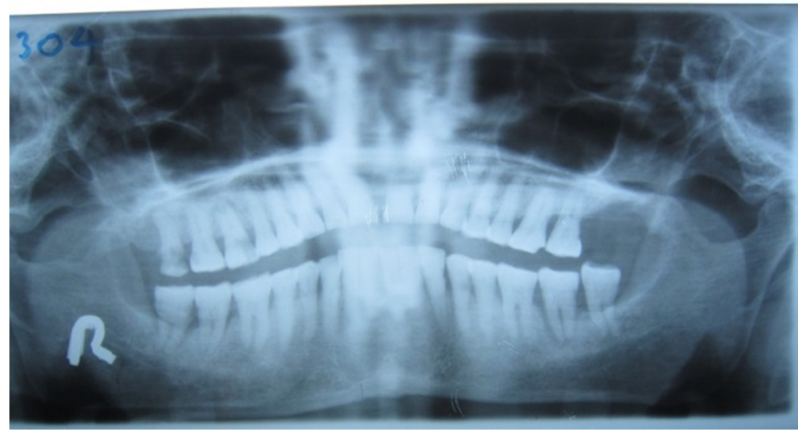

Figure 3. Head tipped-up. Hard Palate is covering the roots of maxillary teeth and 'reverse smile' indicative of head tipped to up. And both TemporoMandibular Joints (TMJs) are not included in the image. Note handling artifacts over the ROI.

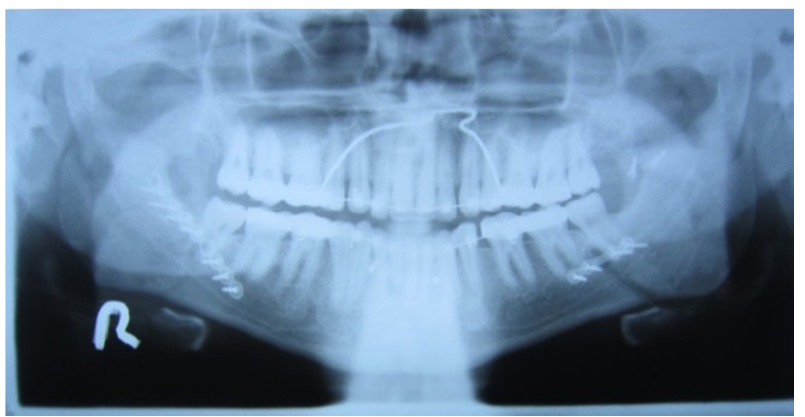

Figure 4. The slumped cervical spine shadow is appearing as a pyramid shaped opacity centered at the lower half of the film.

\section{Discussion}

In our study all possible errors in panoramic radiography including handling and processing were included since the radiographs were produced from a conventional manual panoramic machine with a manual film processor. The study was conducted on conventional panoramic radiographs directly viewed on an illuminator since conventional systems are common in Sri Lanka although the digital systems are increasingly being used in dentistry all over the world.

The present study assessed the variety of unique errors related to conventional panoramic radiographic techniques which would cause degradation of image quality by means of a clinical audit. Malpositioning was the most frequent error encountered in OPG procedures in DHTP. In the present study there were $88 \%$ of positioning errors and this finding is in agreement with most of the related studies $[1,7,9,11,12]$. It is a very high value although the films were not rejected due to any of these positioning errors. Tongue was not placed against the roof of the mouth; hence a dark radiolucent band over the roots of the upper teeth was resulted in 86 (34\%) images as the most apparent positioning error and this is in agreement with results some previous studies [9]. In contrast to previous studies, among patient positioning errors slumped cervical spine was resulted in $54(20 \%)$ of radiographs as the second most frequent error.

There is an impact of the performance and skill of the radiographer directly on the quality of panoramic radiograph. Patient positioning should be done with great care by radiographers [9]. As seen in most images we can conclude that patients were not instructed well about how they should be positioned during the exposure. Hence, expending time in patient preparation, patient positioning and good communication with patients as well as the proper use of patient positioning apparatus as suggested by previous researches as well could decrease the number of positioning errors [9].

Film handling and processing errors were the second most common error category resulted in the present study. There were finger prints, scratch marks, fixer and developer stains on the radiographs as frequent errors. Finger print marks appearing on the ROI was the most apparent error determining the poor handling of panoramic radiographs. Generally the technique for processing a radiograph is important for occurrence of errors related to film processing and handling [13, 14]. The use of manual techniques in processing radiographs could be the reason for this significance frequency of processing and handling errors resulted compared to other similar studies have used digital system where they have used digital processing facility.

Although errors in patient preparation were resulted in the present study, it was not the cause of repetition of the radiographs whereas though less occurrence of over or underexposure in which all the radiographs had exposure errors were repeated. This is mainly due to preparation errors were not totally affect the image quality although it diminishes diagnostic quality to some extent. But however, the presence of exposure errors have totally affected the images by reducing overall contrast of the radiographs by eliminating better identification of teeth and their surrounding structures on the radiograph. Thus all the images which affected by exposure errors were repeated.

In conclusion, frequencies of errors encountered on conventional panoramic radiographs were significantly higher in the present study. Malpositioning was resulted as the most frequent error followed by film handling and processing errors. It is reasonable to conclude that operator performance and skills would be beneficial greatly to reduce patient positioning and preparation artifacts while using digital or automated systems for reducing film handling and processing artifacts. 


\section{References}

[1] Choi BR, Choi DH, Huh KH, Yi WJ, Heo MS, Choi SC, et al. Clinical image quality evaluation for panoramic radiography in Korean dental clinics. Imaging Sci Dent. 2012; 42 (3): 18390 .

[2] Whitley, A. Stewart; Sloane, Charles; Hoadley, Graham; Moore, Adrian D.; Alsop CW. Clark's Positioning in Radiography. Vasa. 2005; 126.

[3] Eric whites, Churchill Livingstone Elsevier, Essentials of dental radiography and radiology, dental panoramic radiography $\left(4^{\text {th }}\right.$ edn), Philadelphia, USA, 2007.

[4] Haring JI, Howerton LJ, Panoramic radiography, Dental radiography principles and techniques $\left(3^{\text {rd }} \mathrm{edn}\right)$, Elsevier, India, P A: Saunders, 2006, p p 304-322.

[5] Jayasinghe R. D., Oral radiography and radiology for dental students (2012), dental panoramic radiography ( ${ }^{\text {st }}$ edn), Sri Lanka.

[6] Langland O. E, Sippy F. H., Morris C. R., and Langlais R. P., Principles and Practice of Panoramic Radiology, W B Saunders, London, UK, 2nd edition, 1992.

[7] Peretz B, Gotler M. and Kaffe I, Common Errors in Digital Panoramic Radiographs of Patients with Mixed Dentition and Patients with Permanent Dentition, International Journal of
Dentistry, Volume 2012 (2012), Article ID 584138, doi:10.1155/2012/584138 Research Article.

[8] William C. Scarfe, Gail F. Williamson, Practical Panoramic Radiography, Revised July 25, 2007, Available: http://media.dentalcare.com/media/enus/education/ce71/ce71.pdf

[9] Dhillon M, Raju SM, Verma S, Tomar D, Mohan RS, Lakhanpal M, et al. Positioning errors and quality assessment in panoramic radiography. Imaging Sci Dent. 2012;42 (4): 207-12.

[10] Manufacture's guidelines, Planmeca Proline XC radiographic machine, asentajankatu6, 00880 Helsinki, Finland.

[11] Sabarudin A, Tiau YJ. Image quality assessment in panoramic dental radiography: a comparative study between conventional and digital systems. Quant Imaging Med Surg. 2013; 3(1): 438.

[12] Brezden NA, Brooks SL. Evaluation of panoramic dental radiographs taken in private practice. Oral Surg Oral Med Oral Pathol. 1987; 63: 617-621.

[13] Langland OE, Langlais RP, Preece JW. Principles of Dental Imaging, 2nd ed. Philadelphia: Lippincott Williams \& Wilkins; 2002: 219-258.

[14] Kaviani F, Johari M, Esmaeili F. Evaluation of common errors of panoramic radiographs in tabriz faculty of dentistry. $J$ Dent Res Dent Clin Dent Prospects [Internet]. 2008; 2 (3): 99-101. 